\title{
European neonatal intensive care nursing research priorities: an e-Delphi study
}

\author{
Joke M Wielenga, ${ }^{1}$ Lyvonne N Tume, ${ }^{2}$ Jos M Latour, ${ }^{3,4,5}$ Agnes van den Hoogen ${ }^{6}$
}

\begin{abstract}
${ }^{1}$ Department of Intensive Care Neonatology, Emma Children's Hospital/Academic Medical Centre, Amsterdam, The Netherlands

${ }^{2} \mathrm{PICU}$ and Children's Nursing Research Unit, Alder Hey Children's NHS FT, Liverpool UK and University of Central Lancashire, Preston, UK ${ }^{3}$ Faculty of Health and Human Sciences, School of Nursing and Midwifery, Plymouth University, Plymouth, UK ${ }^{4}$ Faculty of Health Sciences, School of Nursing and Midwifery, Curtin University, Perth, Western Australia, Australia

${ }^{5}$ Department of Paediatrics, Intensive Care Neonatology, Erasmus MC-Sophia

Children's Hospital, Rotterdam, The Netherlands

${ }^{6}$ Department of Intensive Care Neonatology, Wilhelmina Children's Hospital, University Medical Centre Utrecht, Utrecht, The Netherlands
\end{abstract}

\section{Correspondence to}

Dr Joke M Wielenga, Department of IC Neonatology, Emma Children's Hospital/ Academic Medical Centre, PO Box 22660, Amsterdam 1100 DD, The Netherlands; j.wielenga@amc.uva.nl

Received 2 June 2014 Revised 22 August 2014 Accepted 7 September 2014 Published Online First 26 September 2014

\section{ABSTRACT \\ Objective This study aimed to identify and prioritise neonatal intensive care nursing research topics across Europe using an e-Delphi technique.}

Design An e-Delphi technique with three questionnaire rounds was performed. Qualitative responses of round one were analysed by content analysis and research statements were generated to be ranged on importance on a scale of 1-6 (not important to most important).

Setting Neonatal intensive care units (NICUs) in 17 European countries.

Population NICU clinical nurses, managers, educators and researchers $(n=75)$.

Intervention None.

Main outcome measures A list of 43 research statements in eight domains.

Results The six highest ranking statements $(\geq 5.0$ mean score) were related to prevention and reduction of pain (mean 5.49; SD 1.07), medication errors (mean 5.20; SD 1.13), end-of-life care (mean 5.05; SD 1.18), needs of parents and family (mean 5.04; SD 1.23), implementing evidence into nursing practice (mean 5.02; SD 1.03), and pain assessment (mean 5.02; SD 1.11). The research domains were prioritised and ranked: (1) pain and stress; (2) family centred care; (3) clinical nursing care practices; (4) quality and safety; (5) ethics; (6) respiratory and ventilation; (7) infection and inflammation; and (8) professional issues in neonatal intensive care nursing.

Conclusions The results of this study might support developing a nursing research strategy for the nursing section of the European Society of Paediatric and Neonatal Intensive Care. In addition, this may promote more European researcher collaboratives for neonatal nursing research.

\section{INTRODUCTION}

Neonatal intensive care nursing needs strategic directions and a common goal for strengthening and prioritising their nursing practice. WHO formulated the goals of improved health outcomes through the provision of competent, culturally sensitive, and evidence-based neonatal nursing and midwifery services. ${ }^{1}$ A way to achieve these goals is through research initiatives. In addition, nurses, midwifes and parents can be involved in this process to meet their needs, and to encourage adhering to the cornerstone of collaborative action.

If researchers do not know about the most important problems affecting neonatal intensive care (as described by neonatal intensive care unit (NICU) nurses across Europe), then research may be directed in non-priority areas. Furthermore, research priorities are constantly dynamic entities

\section{What is already known on this topic}

- Both adult and paediatric intensive care have defined their research priorities.

- Neonatal intensive care unit nursing research priorities have never been identified.

\section{What this study adds}

- Neonatal intensive care nurses have identified fundamental clinical nursing care issues as a priority research area.

- The identified neonatal intensive care nursing research priorities provide a roadmap for future collaborative research efforts.

that change over time and differ culturally. ${ }^{2}$ It is therefore crucial to determine neonatal intensive care nursing research priorities within Europe.

The European Society of Paediatric and Neonatal Intensive Care (ESPNIC) is a community of paediatric and neonatal intensive care physicians and nurses who share a common goal of promoting and advancing care through research and education. ${ }^{3}$ ESPNIC has restructured and established new themed sections to support these activities. ${ }^{2}$ The nurse science section intends to establish a NICU nursing research agenda within Europe and develop a plan for future collaborative NICU nursing research activities. To achieve this, our present explorative and descriptive study aimed to identify NICU nursing research topics and prioritise the identified topics as defined by European NICU nurses.

\section{METHODS}

We performed a modified three-round e-Delphi study. The e-Delphi technique is a structured process distributing a series of questionnaires during several rounds to gather information and set priorities or gain consensus regarding a specific issue. ${ }^{45}$ The Delphi technique allows the inclusion of a large number of individuals across diverse geographical locations without physically meeting them. To date, the Delphi technique is often conducted via online web surveys, offering a number of advantages as they are quick to set up, relatively low cost and provide high level of data security. ${ }^{6}$ Systematic feedback, structured information flow, and iteration and anonymity are the main characteristics of a Delphi technique. ${ }^{7}$ Systematic feedback of panel members' 
responses takes place in between rounds by informing individual experts about the group opinions. Iteration takes place by presenting feedback via a certain number of rounds. ${ }^{5}$ The performed Delphi method is shown in figure 1.

\section{Participants}

The study sample included NICU clinical nurses, managers, educators and researchers from 17 European countries. It was aimed to generate a representative sample of eight nurses with various positions per country (two clinical practice neonatal nurses, two nurses in NICU education, two NICU nurse managers and two neonatal research nurses). The inclusion criterion was that nurses needed to work in a NICU setting; no minimal years of experience was defined. Exclusion criteria were nurses working in paediatric wards, paediatric intensive care and not taking care of critically ill newborns and infants on a regular basis. Contact details (names and email addresses only) were obtained through the ESPNIC nursing membership registry, through professional contacts and by searching the worldwide web. If less than eight nurses per country were identified, we asked nurses to provide contact details of colleagues in their country to reach the predetermined number of eights nurses per country. If we received more than eight responses per country, all respondents were invited as we did not want to exclude motivated participants who had already been contacted by a country lead. The recruitment strategy elicited 80 potential respondents from 17 European countries at the beginning, of which 75 (94\%) responded to round one. Participants were informed about the voluntary nature of the study, the need for ongoing participation in three Delphi rounds and informed consent was assumed by completion of the questionnaires. Personal data characteristics were retained to determine
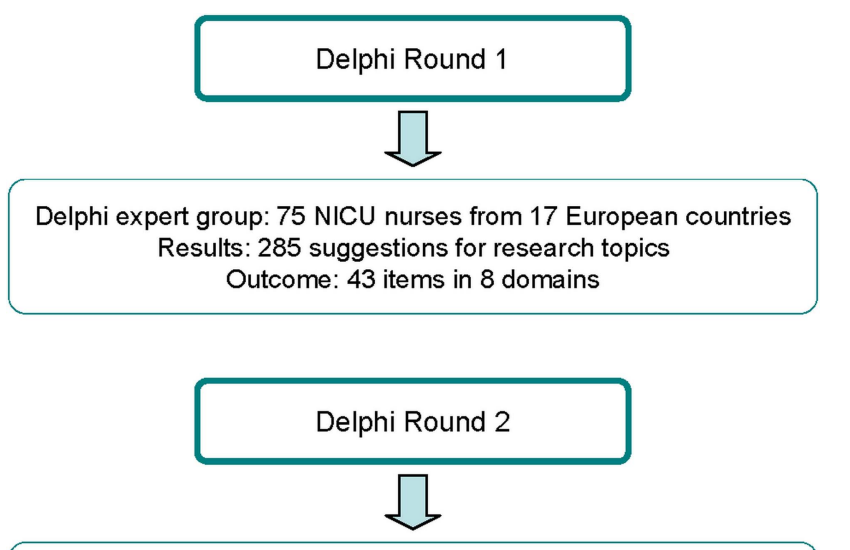

Delphi expert group: 68 NICU nurses from 17 European countries Outcome: Group mean scores (and SD) of 43 items

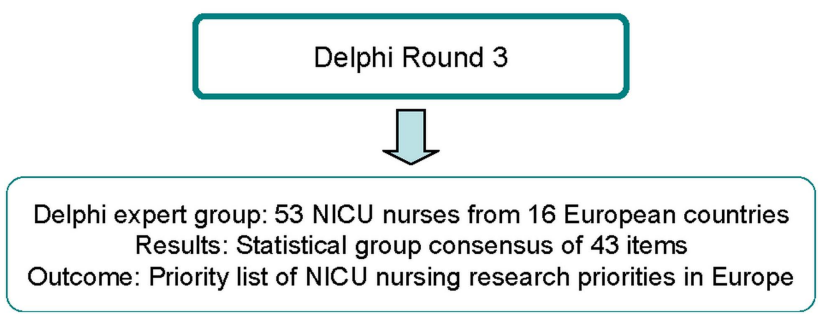

Figure 1 NICU Delphi study flowchart. NICU, neonatal intensive care unit. response rates and link findings to nursing roles and countries with all information stored on a secure password protected database. To maximise the response rate and decrease possible attrition between the three Delphi rounds, the consented 75 nurses received the questionnaire of all three rounds and three reminders per round.

\section{Questionnaire}

The e-Delphi study used three questionnaires for the three consecutive rounds. The first round was a qualitative questionnaire with one question to list a minimum of three and maximum of five priority research topics for NICU nursing. Two researchers (JMW and $\mathrm{AvdH}$ ) independently performed content analysis on the answers; any disagreement was discussed and agreement was reached through discussion. This was then checked for validity by two other researchers (LNT and JML). The research statements were clustered into thematic domains according to the content and number of suggestions using an analysis framework. ${ }^{8} 9$ The content analysis generated a list of research statements and domains for round two. If the number of research statements relating to a specific topic was high, we agreed this warranted a domain area of its own. The choice to add statements regarding parent education, discharge planning, breast feeding and kangaroo mother care to the domain clinical nursing care practices instead of the domain family centred care is based on the fact that in many countries this is part of daily clinical care practice whereas family centred care is not yet uniformly practised across Europe. For round two of the study, participants were asked to rank these statements and domains on a 6-point scale ( 1 not important to 6 extremely important). In round three, the questionnaire contained the same research statements and domains including the group mean scores of the previous round per statement and domain. Data collection of the three rounds e-Delphi questionnaires was completed between September 2012 and February 2013.

\section{Statistical analysis}

Mean and SDs of the round two and three responses were calculated. In the final analysis of round three, the statements were ranked on importance by calculating the means and SD. Cohen's d was used to complement the $t$ test by providing information on the relative magnitude of the effect size comparing the responses between rounds two and three. The interpretation of the Cohen's d (standardised mean difference) is: 0.2 small effect, 0.5 medium effect and $>0.8$ large effect. ${ }^{10}$ The paired $t$ test was used to calculate difference between rounds two and three. Significance level was set at $<0.05$. The importance of the statements was determined by the highest mean and smallest SD. A lead individual NICU nurse expert per country provided the translation of the questionnaires. A forward translation, with a double check with the translators in case of lack of clarity, was used. European regions were categorised for analysis using the definition in the ETHICUS study. ${ }^{1}{ }^{11}$ SurveyMonkey Gold version was selected to administer the e-Delphi questionnaires and the data analysis was performed using IBM SPSS V.20 software.

Ethical approval was granted from the Institutional Review Board approval of the University Medical Centre Utrecht, The Netherlands (protocol number 12/147).

\section{RESULTS}

Of the 80 nurses invited to participate, 75 (94\%) nurses from 17 European countries agreed to participate. The dispersion of the participants varied per country with one participating NICU 
nurse from Finland, Greece, and Portugal to 10 NICU nurses in the UK. The response rates of consecutive rounds were: round two 68/75 (90.6\%) and round three 53/75 (70.6\%) (figure 1). Variation was seen in the number of nurses and roles per country because some countries had no nursing roles in NICU education or research. In the UK, more than eight nurses started in round one. This was because in our over-recruitment strategy, all invited NICU nurses actually responded and participated. We did not want to exclude these motivated nurses who had already been contacted by the country lead. The characteristics of the respondents remained similar over the three rounds (table 1).

Totally, 285 research topics were provided in round one and content analysis revealed 43 research statements divided in eight domains. The 43 statements ranged from the lowest mean score of 4.05 (SD 1.21) to the highest mean score 5.18 (SD 1.19) in rounds two and three (table 3). The eight research domains identified were: pain and stress (mean 5.18; SD 1.19), family centred care (mean 4.84; SD 1.29), clinical nursing care practices (mean 4.82; SD 1.16), quality and safety (mean 4.78; SD 1.15), ethics (mean 4.64; SD 1.16), respiratory and ventilation (mean 4.44; SD 1.10), infection and inflammation (mean 4.16; SD 1.24), and professional issues in neonatal intensive care nursing (mean 4.05; SD 1.21). There was no significant change in mean scores on domain level between rounds two and three (table 2).

On the level of individual statements, five statements, related to environmental factors and neonatal development, neonatal temperature, palliative care pathways, sepsis management, and nursing education and training, scored in round three statistically significantly lower compared with round two. One statement on identifying interventions to implement evidence into practice scored statistically significantly higher in round three compared with round two (table 2). In round three, six statements reached a mean score of $\geq 5.0$. These were related to interventions to prevent or reduce pain, best practice for pain assessment, reducing medication errors, end-of-life care, supporting the needs of parents and family members and implementing evidence-based practice (table 3 ).

No significant differences were found in the ranking of research priorities between European regions (table 4).

\begin{tabular}{|c|c|c|c|}
\hline & $\begin{array}{l}\text { Round } 1 \\
\mathrm{n}=75\end{array}$ & $\begin{array}{l}\text { Round } 2 \\
n=68\end{array}$ & $\begin{array}{l}\text { Round } 3 \\
n=53\end{array}$ \\
\hline Female $(\%)$ & 87.7 & 72.1 & 75.5 \\
\hline Age (years); mean (SD) & $44.9(10.1)$ & $44.0(9.0)$ & $45.1(9.5)$ \\
\hline NICU experience (years); mean (SD) & $17.7(9.0)$ & $17.7(7.8)$ & $18.1(7.9)$ \\
\hline \multicolumn{4}{|l|}{ Main nursing role } \\
\hline Clinical (\%) & 34.2 & 27.9 & 28.3 \\
\hline Education (\%) & 19.2 & 26.5 & 24.5 \\
\hline Research (\%) & 24.7 & 19.1 & 22.6 \\
\hline Management (\%) & 20.5 & 20.6 & 18.9 \\
\hline Missing (\%) & 1.4 & 5.9 & 5.7 \\
\hline \multicolumn{4}{|l|}{ Unit type } \\
\hline NICU (\%) & 67.1 & 52.9 & 64.2 \\
\hline PICU-NICU combined (\%) & 21.9 & 16.2 & 17.0 \\
\hline Missing (\%) & 11.0 & 30.9 & 18.9 \\
\hline
\end{tabular}

\section{DISCUSSION}

Until now, NICU research priorities had never been identified. European NICU nurses have prioritised pain and stress as well as fundamental clinical nursing care issues for critically ill newborns and their families (family centred care) within NICU nursing practice. Quality and safety, ethics, respiratory and ventilation, infection and inflammation and organisational and professional issues were also identified as priority research areas. Other studies of research priorities in critical care nursing have identified similar findings. ${ }^{12-18}$ Many of these have also used Delphi-type techniques to generate research priorities. A European adult intensive care study identified research priorities that related to organisational aspects of clinical intensive care practice and organ-system support. ${ }^{16}$ In an Australian and New Zealand Delphi study of paediatric intensive care unit (PICU) nursing research priorities, top priorities included patient issues related to neurological care, pain/sedation/comfort, best practice at the end of life and ventilation strategies, as well as two priorities related to professional issues about nurses' stress/burnout and professional development needs. ${ }^{14}$ In a recent study undertaken with PICU nurses from Europe in 2013, top priorities were related to issues such as end-of-life care, sustaining treatment, prevention of pain and reducing healthcare-associated infections, but also education, staffing and implementing evidence into practice. ${ }^{13}$

Our study showed that on the level of individual statements, five statements, related to environmental factors and neonatal development, scored in round three statistically significantly lower compared with round two. The top nursing research priorities identified in our study relate to prevention and reduction of pain, medication errors, end-of-life care, the needs of parents and family, implementing evidence into nursing practice and pain assessment. Some individual NICU nurse researchers are already conducting research in several of these research areas. ${ }^{19-25}$ They either work nationally or internationally, such as a European study on end-of-life decision making practices supported by ESPNIC or a national study on end-of-life practices in NICU. ${ }^{19}{ }^{20}$ Other nurse researchers are active in pain and stress management in neonates. ${ }^{21-23}{ }^{26-31}$ Other areas of active NICU nursing research are in staffing levels, education, parents and clinical issues. ${ }^{30} 31$

The research priorities generated in this study do not necessarily reflect a lack of research in these areas, but rather may represent a lack of effective implementation of research evidence into clinical nursing practice. Or indeed that even despite the research conducted in NICU nursing, nurses still believe more research is needed. This is the case for a number of our identified research priorities. Systematic reviews are available on breast milk, venepuncture, oral sucrose and glucose, kangaroo care and non-pharmacological pain management in NICU. ${ }^{26-31}$ Yet, this evidence may not have been translated into nursing practice The research priorities identified in our study could be associated with the lack of interventions to implement evidence into NICU nursing practice. A statement related to this shortcoming scored statistically significantly higher in round three compared with round two.

The findings of this study may promote neonatal nurse researchers across Europe to collaborate more on priority areas and establish new collaboratives focusing on these priority topics. There is some concern that Delphi studies identifying research priorities have not impacted on actual research outputs, but if used within a framework supported by an organisation such as ESPNIC, they are more likely to be effective. ${ }^{32}$ Establishing research priorities is advocated for helping 
Table 2 Results of domains and statements of rounds two and three

\begin{tabular}{|c|c|c|c|c|}
\hline Domains and statements & $\begin{array}{l}\text { Round } 2 \\
\text { mean (SD) }\end{array}$ & $\begin{array}{l}\text { Round } 3 \\
\text { mean (SD) }\end{array}$ & Cohen's d & p Value \\
\hline 1. Pain and stress & $4.96(1.26)$ & $5.18(1.19)$ & -0.18 & 0.19 \\
\hline Identifying effective interventions to prevent or reduce pain or stress & $5.11(1.52)$ & $5.49(1.07)$ & -0.29 & 0.21 \\
\hline Identifying best practices for pain assessment & $5.07(1.13)$ & $5.02(1.11)$ & 0.05 & 0.83 \\
\hline Identifying pain and/or stress guidelines & $4.84(1.02)$ & $4.76(1.05)$ & 0.08 & 0.82 \\
\hline 2. Family centred care & $4.86(1.44)$ & $4.84(1.29)$ & 0.10 & 0.76 \\
\hline Identifying strategies to support the needs of parents and family members & $5.05(1.13)$ & $5.04(1.23)$ & 0.01 & 0.89 \\
\hline Identifying and evaluating strategies to support parental attachment & $4.94(1.09)$ & $4.85(1.37)$ & 0.07 & 0.56 \\
\hline Identifying best practices for the implementation of family centred care & $4.69(1.25)$ & $4.80(1.21)$ & -0.09 & 0.91 \\
\hline Evaluating the role and involvement of parents in the care of their infant & $4.83(1.20)$ & $4.78(1.20)$ & 0.04 & 0.43 \\
\hline Evaluating developmental care & $4.76(1.06)$ & $4.75(1.14)$ & 0.01 & 1.00 \\
\hline Evaluating the effect of environmental factors on neonatal development & $4.81(1.09)$ & $4.65(1.11)$ & 0.15 & 0.03 \\
\hline Evaluating individual care aspects (sleep, positioning, body language) of developmental care & $4.66(1.19)$ & $4.60(1.15)$ & 0.06 & 0.32 \\
\hline Evaluating the effectiveness of NIDCAP and NIDCAP strategies & $4.61(1.16)$ & $4.31(1.18)$ & 0.26 & 0.09 \\
\hline 3. Clinical nursing care practices & $4.71(1.39)$ & $4.82(1.16)$ & -0.09 & 0.47 \\
\hline Improving the care and parental education of chronically ill neonates requiring long term care & $4.77(1.30)$ & $4.76(1.22)$ & 0.01 & 0.94 \\
\hline Nursing management of very low birthweight infants & $4.53(1.32)$ & $4.75(1.19)$ & -0.18 & 0.07 \\
\hline Improving nutrition in preterm and sick term infants & $4.74(1.33)$ & $4.67(1.19)$ & 0.06 & 0.88 \\
\hline Identifying best practice in the management of invasive lines and catheters & $5.02(1.09)$ & $4.65(1.16)$ & 0.33 & 0.08 \\
\hline Identifying best practices in enteral feeding & $4.38(1.15)$ & $4.60(1.20)$ & -0.19 & 0.20 \\
\hline Identifying best practices breast feeding & $4.20(1.23)$ & $4.53(1.10)$ & -0.28 & 0.08 \\
\hline Identifying and evaluating interventions to improve skin and wound care in neonates & $4.60(1.43)$ & $4.45(1.21)$ & -0.14 & 1.00 \\
\hline Identifying and implementing best practices in discharge planning & $4.64(1.30)$ & $4.45(1.43)$ & 0.14 & 0.60 \\
\hline Identifying the best care practices for infants with neurological problems & $4.57(1.41)$ & $4.44(1.12)$ & 0.10 & 0.72 \\
\hline Improving advanced life support strategies to improve patient outcomes & $4.65(1.18)$ & $4.44(1.12)$ & 0.18 & 0.46 \\
\hline Identifying the best care practices for surgical infants & $4.45(1.37)$ & $4.24(1.18)$ & 0.16 & 0.38 \\
\hline Identifying and implementing strategies to promote kangaroo mother (skin-to-skin) care & $4.24(1.27)$ & $4.20(1.39)$ & 0.03 & 0.80 \\
\hline Evaluating strategies for regulation of neonatal temperature & $4.95(1.18)$ & $4.13(1.09)$ & 0.72 & $<0.01$ \\
\hline Evaluating routine nursing care procedures & $4.48(1.18)$ & $4.00(1.37)$ & 0.38 & 0.17 \\
\hline 4. Quality and safety & $4.72(1.33)$ & $4.78(1.15)$ & -0.05 & 0.84 \\
\hline Identifying and evaluating strategies to reduce medication errors & $5.03(1.05)$ & $5.20(1.19)$ & -0.15 & 0.35 \\
\hline Identifying safe medication administration practices & $4.61(1.23)$ & $4.96(1.19)$ & -0.29 & 0.07 \\
\hline Improving patient safety and patient outcomes & $4.79(1.23)$ & $4.78(1.03)$ & 0.01 & 0.74 \\
\hline Improving healthcare team communication and collaboration & $4.73(1.17)$ & $4.78(1.17)$ & -0.04 & 0.44 \\
\hline Identifying and implementing a safe working environment for staff & $4.68(1.11)$ & $4.36(1.21)$ & 0.28 & 0.10 \\
\hline 5. Ethics & $4.44(1.45)$ & $4.64(1.16)$ & -0.15 & 0.49 \\
\hline Improving end-of-life care for neonates and their families & $4.73(1.21)$ & $5.05(1.18)$ & -0.27 & 0.14 \\
\hline Exploring the role of parents in ethical decision making & $4.90(1.27)$ & $4.95(1.35)$ & -0.04 & 0.94 \\
\hline Developing palliative care pathways for neonates & $5.24(1.04)$ & $4.78(1.10)$ & 0.43 & 0.03 \\
\hline 6. Respiratory and ventilation & $4.29(1.44)$ & $4.44(1.10)$ & -0.12 & 0.46 \\
\hline Identifying best practices in the care of non-invasive ventilation in infants & $4.97(1.07)$ & $4.85(1.21)$ & 0.11 & 0.92 \\
\hline Identifying best practices in the care of the mechanically ventilated infant & $4.92(1.23)$ & $4.65(1.31)$ & 0.21 & 0.38 \\
\hline 7. Infection and inflammation & $4.07(1.54)$ & $4.16(1.24)$ & 0.01 & 0.87 \\
\hline Evaluating infection prevention strategies & $5.11(1.17)$ & $4.78(1.29)$ & 0.27 & 0.57 \\
\hline Identifying and evaluating interventions to monitor and reduce hospital-associated infections & $4.96(1.19)$ & $4.71(1.20)$ & 0.21 & 0.21 \\
\hline Evaluating sepsis management and care to improve outcomes & $5.05(1.04)$ & $4.36(1.50)$ & 0.60 & 0.03 \\
\hline 8. Professional issues in NICU nursing & $4.14(1.50)$ & $4.05(1.21)$ & 0.07 & 0.81 \\
\hline Identifying interventions to implement evidence into NICU nursing practice & $4.59(1.41)$ & $5.02(1.03)$ & -0.35 & 0.03 \\
\hline Identifying strategies to reduce stress and improve performance in NICU nursing & $4.60(1.30)$ & $4.85(1.11)$ & -0.21 & 0.10 \\
\hline Evaluating nursing education and training strategies & $5.08(0.83)$ & $4.64(1.11)$ & 0.45 & 0.03 \\
\hline $\begin{array}{l}\text { Evaluating the impact of the changing NICU workforce on patient outcomes (advanced nurse practice roles, physician } \\
\text { assistants, etc) }\end{array}$ & $4.53(1.26)$ & $4.55(1.10)$ & -0.02 & 0.40 \\
\hline Identifying optimal nurse staffing levels & $5.00(0.98)$ & $4.49(1.25)$ & 0.45 & 0.10 \\
\hline
\end{tabular}

Scoring on a 6-point scale.

NICU, neonatal intensive care unit; NIDCAP, Newborn Individualized Developmental Care and Assessment Program.

researchers gain research funding aligned with European evidence needs. $^{1427283334}$ The results of this study are intended to develop a European nursing research agenda and a future roadmap with the support of ESPNIC. ${ }^{13}$ These priorities however are dynamic and will change over time, and thus need revisiting in the future. 
Table 3 Top 20 ranking NICU research statements

\begin{tabular}{|c|c|c|}
\hline Research statement & & Mean (SD) \\
\hline 1 & Identifying effective interventions to prevent or reduce pain or stress & $5.49(1.07)$ \\
\hline 2 & Identifying and evaluating strategies to reduce medication errors & $5.20(1.13)$ \\
\hline 3 & Improving end-of-life care for neonates and their families & $5.05(1.18)$ \\
\hline 4 & Identifying strategies to support the needs of parents and family members & $5.04(1.23)$ \\
\hline 5 & Identifying interventions to implement evidence into NICU nursing practice & $5.02(1.03)$ \\
\hline 6 & Identifying best practices for pain assessment & $5.02(1.11)$ \\
\hline 7 & Identifying safe medication administration practices & $4.96(1.19)$ \\
\hline 8 & Exploring the role of parents in ethical decision making & $4.95(1.35)$ \\
\hline 9 & Identifying strategies to reduce stress and improve performance in NICU nursing & $4.85(1.11)$ \\
\hline 10 & Identifying best practices in the care of non-invasive ventilation in infants & $4.85(1.21)$ \\
\hline 11 & Identifying and evaluating strategies to support parental attachment & $4.85(1.37)$ \\
\hline 12 & Identifying best practices for the implementation of family centred care & $4.80(1.21)$ \\
\hline 13 & Improving patient safety and patient outcomes & $4.78(1.03)$ \\
\hline 14 & Developing palliative care pathways for neonates & $4.78(1.10)$ \\
\hline 15 & Improving healthcare team communication and collaboration & $4.78(1.17)$ \\
\hline 16 & Evaluating the role and involvement of parents in the care of their infant & $4.78(1.20)$ \\
\hline 17 & Evaluating infection prevention strategies & $4.78(1.29)$ \\
\hline 18 & Identifying pain and/or stress guidelines & $4.76(1.05)$ \\
\hline 19 & Improving the care and parental education of chronically ill neonates requiring longterm care & $4.76(1.22)$ \\
\hline 20 & Evaluating developmental care & $4.75(1.14)$ \\
\hline
\end{tabular}

Some limitations of our study need to be recognised. The first is that we did not examine NICU parent's perspectives on NICU research priorities or indeed other healthcare professionals involved in the care of these infants. Second, there was considerable variation in the number of nurses per country and some European countries were not represented. All efforts were made to have a sample representing all European countries; however, this was not achievable for all countries. Another potential bias within this study is that due to the way that NICU is delivered within Europe, some NICUs are combined with paediatric intensive care. Therefore, there may be some 'overlapping' of the NICU nursing study participants with that of paediatric intensive care nurses. Finally, using the ESPNIC registry as a starting point could imply that the results reflect the opinion of the ESPNIC members. However, the experts were asked to identify nurses and email addresses in various roles and across various units and participants in this study were asked to provide their opinion based on their own NICU experience and expertise. In addition, we were not able to analyse research priorities by nursing role because some countries did not have research nurses or education nursing roles in NICUs and thus analysing only small numbers of these respondents would introduce bias into the countries that have these roles.

The main strength of this e-Delphi study is that it was electronic in nature. Electronic surveys enable more rapid responses, more rapid data analysis, less attrition between survey rounds and reduce costs. ${ }^{6}$ Furthermore, having local translations of the survey meant that it did not restrict it to only English-speaking nurses, which has been a limitation of other studies. ${ }^{16}$

A primary goal for the ESPNIC nurse science section is initiating research programmes. It goes without saying that a Delphi study focusing on establishing research priorities is a good starting point to reach this goal. In conclusion, eight NICU nursing priority research domains were identified. The findings of this study may promote neonatal nurse researchers across Europe to collaborate more on priority areas and establish new research

Table 4 Comparison of research domains per European region, round three

\begin{tabular}{|c|c|c|c|c|c|}
\hline Research domain & $\begin{array}{l}\text { Overall } \\
\text { mean (SD) } \\
n=55\end{array}$ & $\begin{array}{l}\text { Northern Europe } \\
\text { mean (SD) } \\
n=27\end{array}$ & $\begin{array}{l}\text { Central Europe } \\
\text { mean (SD) } \\
n=20\end{array}$ & $\begin{array}{l}\text { Southern Europe } \\
\text { mean (SD) } \\
n=8\end{array}$ & p Value \\
\hline Pain and stress & $5.18(1.19)$ & $5.07(1.30)$ & $5.15(1.23)$ & $5.62(0.52)$ & 0.927 \\
\hline Family centred care & $4.84(1.29)$ & $4.89(1.01)$ & $4.90(1.33)$ & $4.50(2.00)$ & 0.258 \\
\hline Clinical nursing care practices & $4.82(1.16)$ & $4.67(1.33)$ & $4.82(1.16)$ & $5.13(0.64)$ & 0.816 \\
\hline Quality and safety & $4.78(1.15)$ & $4.67(1.18)$ & $4.90(1.07)$ & $5.38(0.74)$ & 0.605 \\
\hline Ethics & $4.64(1.16)$ & $4.48(1.19)$ & $4.70(1.26)$ & $5.00(0.76)$ & 0.115 \\
\hline Respiratory and ventilation & $4.44(1.10)$ & $4.15(0.82)$ & $4.55(1.43)$ & $5.13(0.64)$ & 0.082 \\
\hline Infection and inflammation & $4.16(1.24)$ & $4.19(1.24)$ & $3.95(1.32)$ & $4.63(1.06)$ & 0.897 \\
\hline Professional issues in NICU nursing & $4.05(1.21)$ & $3.85(1.03)$ & $4.30(1.22)$ & $4.13(1.73)$ & 0.159 \\
\hline
\end{tabular}


collaboratives focusing on these priority topics, which in turn may assist in achieving research funding. And including parents in research teams is not an option but rather essential to allow empowerment and involvement of parents in all healthcare activities.

Acknowledgements The authors thank the NICU colleagues for providing their expertise. Our colleagues who helped with the translation of the questionnaires are heartily thanked: Immacolata Dall'Oglio, Gordana Dragosev, Orsola Gawronski, Irene Harth, Petr Jirasek, Stephane Leteurtre and Lurdes Lisboa. The authors also acknowledge the dedication and support of ESPNIC.

Contributors JMW: contributed to the development of the research protocol, data collection, data analysis and interpretation; writing the first draft of the manuscript; approved the final version for submission; agrees to be accountable for all aspects of the work in ensuring that questions related to the accuracy or integrity of any part of the work are appropriately investigated and resolved. LNT and JML: contributed to the development of the research protocol, data collection, data analysis and interpretation; revising drafts of the manuscript; approved the final version for submission; agree to be accountable for all aspects of the work in ensuring that questions related to the accuracy or integrity of any part of the work are appropriately investigated and resolved. AvdH: contributed to the development of the research protocol and applied for ethical approval, data collection, data analysis and interpretation; revising drafts of the manuscript; approved the final version for submission; agrees to be accountable for all aspects of the work in ensuring that questions related to the accuracy or integrity of any part of the work are appropriately investigated and resolved.

Funding This study was partially supported by the European Society of Paediatric and Neonatal Intensive Care (ESPNIC).

Competing interests None.

Ethics approval University Medical Centre Utrecht, The Netherlands (protocol number 12/147).

Provenance and peer review Not commissioned; externally peer reviewed.

\section{REFERENCES}

1 Health Professions Networks, Nursing and Midwifery Office Department of Human Resources for Health World Health Organization. Strategic directions for strengthening nursing and midwifery services-2011-2015. http://www.who.int/ hrh/nursing_midwifery/en/ (accessed 6 May 2014).

2 National Institute of Nursing Research; building the scientific foundation for clinical practice. https://www.ninr.nih.gov/ (accessed 6 May 2014).

3 European Society of Paediatric and Neonatal Intensive Care. http://www. espnic-online.org (accessed 6 May 2014).

4 Keeney S, Hasson F, McKenna H. Consulting the oracle: ten lessons from using the Delphi technique in nursing research. J Adv Nurs 2006;53:205-12.

5 Keeney S, Hasson F, McKenna H. The Delphi technique in nursing and health research. Chichester, UK: Wiley-Blackwell, 2011.

6 Gill FJ, Leslie GD, Grech C, et al. Using a web-based survey tool to undertake a Delphi study: application for nurse education research. Nurse Educ Today 2013;11:1322-8.

7 Dalkey NC. The Delphi method: an experimental study of group opinion. Santa Monica, USA: RAND Corporation, 1969.

8 Pope C, Ziebland S, Mays N. Qualitative research in health care. Analysing qualitative data. BMJ 2000;320:114-16.
9 Creswell JW. Research design: qualitative, quantitative, and mixed methods approaches. 4th edn. Los Angeles: Sage Publications Inc, 2014.

10 Cohen J. Statistical power analysis for the behavioral sciences. New York: Lawrence Erlbaum Associates, 1988.

11 Sprung $\mathrm{CL}$, Cohen SL, Sjokvist $\mathrm{P}$, et al. End-of-life practices in European intensive care units: the Ethicus Study. JAMA 2003;290:790-7.

12 Goldfrad C, Vella K, Bion JF, et al. Research priorities in critical care medicine in the UK. Intensive Care Med 2000;26:1480-8.

13 Tume LN, van den Hoogen A, Wielenga JM, et al. An electronic Delphi study to establish pediatric intensive care nursing research priorities in 20 European countries. Pediatr Crit Care Med 2014;15:e206-13.

14 Ramelet AS, Gill F. A Delphi study on National PICU nursing research priorities in Australia and New Zealand. Aust Crit Care 2012;25:41-57.

15 Imhof L, Abderhalden C, Cignacco E, et al. Swiss Research Agenda for Nursing (SRAN): the development of an agenda for clinical nursing research in Switzerland. Pflege 2008;21:252-61.

16 Blackwood B, Albarran JW, Latour JM. Research priorities of adult intensive care nurses in 20 European countries: a Delphi study. J Adv Nurs 2011;67:550-62.

17 Daly J, Chang EM, Bell PF. Clinical nursing research priorities in Australian critical care: a pilot study. J Adv Nurs 1996;23:145-51.

18 Funk M. Research priorities in critical care nursing. Focus Crit Care 1989;16:135-8.

19 Devictor DJ, Latour JM, EURYDICE II study group. Forgoing life support: how the decision is made in European pediatric intensive care units. Intensive Care Med 2011;37:1881-7.

20 Catlin A, Carter B. State of the art. Creation of a neonatal end-of-life palliative care protocol. J Perinatol 2002;22:184-95.

21 Ganzewinkel C Andriessen P. Chronic pain in the neonate: a research design connecting Ancient Delphi to the modern 'Dutch Polder'. JRN 2012;17:262-72.

22 Wielenga JM. Stress and discomfort in the care of preterm infants. A study to the Comfort scale and the Newborn Developmental Care and Assessment Program (NIDCAP®) in a Dutch level III NICU [Thesis]. April 2008.

23 Mainous RO. A Delphi study on categorization of noxious stimuli in the NICU. Adv Neonatal Care 2008;8:135.

24 Latour JM, Hazelzet JA, Duivenvoorden HJ, et al. Perceptions of parents, nurses, and physicians on neonatal intensive care practices. J Pediatr 2010;157:215-20.

25 Cornick P. Nitric oxide education survey - use of a Delphi survey to produce guidelines for training neonatal nurses to work with inhaled nitric oxide. J Neonat Nurs 2006;12:62-8.

26 Shah PS, Herbozo C, Aliwalas LL, et al. Breastfeeding or breast milk for procedural pain in neonates. Cochrane Database Syst Rev 2012;(12):CD004950.

27 Shah VS, Ohlsson A. Venepuncture versus heel lance for blood sampling in term neonates. Cochrane Database Syst Rev 2011;(10):CD001452.

28 Stevens B, Yamada J, Lee GY, et al. Sucrose for analgesia in newborn infants undergoing painful procedures. Cochrane Database Syst Rev 2013;(1):CD001069.

29 Bueno M, Yamada J, Harrison D, et al. A systematic review and meta-analyses of non-sucrose sweet solutions sucrose for pain relief in neonates. Pain Res Manag 2013;18:153-61.

30 Johnston C, Campbell-Yeo M, Fernandes A, et al. Skin-to-skin care for procedural pain in neonates. Cochrane Database Syst Rev 2014;(1):CD008435.

31 Pillai Riddell RR, Racine NM, Turcotte K, et al. Non-pharmacological management of infant and young child procedural pain. Cochrane Database Syst Rev 2011;(10): CD006275.

32 Marshall A. Research priorities for Australian critical care nurses: do we need them? Aust Crit Care 2004;17:142-4, 146, 148-50

33 Franck LS. Research with newborn participants. Doing the right research and doing it right. J Perinat Neonatal Nurs 2005;19:177-86.

34 Dols JD, Bullard K, Gembol L. Research reflections. Setting a nursing research agenda. JONA 2010;40:201-4. 